\title{
Article
}

\section{The Interaction between the LEO Satellite Constellation and the Space Debris Environment}

\author{
Shuyi Ren ${ }^{1,2,3, *(\mathbb{C})}$, Xiaohua Yang ${ }^{4}$, Ronglan Wang ${ }^{1,3}$, Siqing Liu ${ }^{1,3}$ and Xiaojing Sun ${ }^{1,2,3}$ (D) \\ 1 National Space Science Center, Chinese Academy of Sciences, Beijing 100190, China; \\ wangrl@nssc.ac.cn (R.W.); liusq@nssc.ac.cn (S.L.); sunxiaojing17@mails.ucas.ac.cn (X.S.) \\ 2 University of Chinese Academy of Sciences, Beijing 100049, China \\ 3 Key Laboratory of Science and Technology on Environmental Space Situation Awareness, CAS, \\ Beijing 100190, China \\ 4 NO. 31010 Troops, Beijing 100081, China; yxhua3@163.com \\ * Correspondence: syren1996@sina.com
}

check for updates

Citation: Ren, S.; Yang, X.; Wang, R.; Liu, S.; Sun, X. The Interaction between the LEO Satellite Constellation and the Space Debris Environment. Appl. Sci. 2021, 11, 9490. https://doi.org/10.3390/ app11209490

Academic Editor: Rosario Pecora

Received: 15 September 2021

Accepted: 10 October 2021

Published: 13 October 2021

Publisher's Note: MDPI stays neutral with regard to jurisdictional claims in published maps and institutional affiliations.

Copyright: (c) 2021 by the authors. Licensee MDPI, Basel, Switzerland. This article is an open access article distributed under the terms and conditions of the Creative Commons Attribution (CC BY) license (https:/ / creativecommons.org/licenses/by/ $4.0 /)$.

\begin{abstract}
The wide application of satellite constellations in the field of space-based global communications and remote sensing has led to a substantial increase in small-satellite launch plans, a sharp increase in the density of space objects in low-Earth orbit (LEO), and a reduction in available orbit and frequency resources. This will further aggravate the trend of deterioration of the space debris environment. Taking the Starlink constellation as an example, this paper describes the influence of the constellation from the environmental debris flux of the satellite, the evaluation of the number of evasion maneuvers, the change of risk level, the success rate of post mission disposal (PMD) and the growth rate of space objects. The simulation results show that the collision risk of the Starlink constellation is related to the orbital parameters, and the higher success rate of post-mission disposal (PMD) can reduce the collision risk of the constellation. The large constellations increases the growth rate of space objects, and even if all the satellites are disposed of after the mission, the impact of constellations on the space environment can not be offset.
\end{abstract}

Keywords: satellite constellation; space debris; collision probability; risk assessment

\section{Introduction}

The low Earth orbit (LEO) constellation is widely used in space-based global communications and remote sensing. Through the integration of Internet technology and satellite technology, the development of a cloud service platform that continuously updates multi-source data, and the analysis and prediction of earth observation image information based on big data mining and other services will become the focus of the next research [1]. However, large-scale LEO constellations have generated a series of problems for the human space industry, including severe threats to the space debris environment, vicious competition for orbit and frequency resources, and potential risks of international conflicts in dense low-orbit regions. The increase in the number of space objects has begun to threaten the safety of the LEO area. Kessler showed that when the LEO debris density reaches a critical value, it will cause the debris to produce a chain impact effect, rendering the near-Earth space area completely unusable [2]. Due to a large number of satellites in the LEO constellation, the debris generated when passing through the dense area of space targets during the launch process will increase the number of space objects and the collision probability of itself.

In recent years, more and more companies have begun to put forward low-orbit large constellation launch plans. For example, OneWeb plans to build a constellation of 720 satellites at the height of $1200 \mathrm{~km}$ orbit, which are evenly distributed on 18 near-polar orbits [3]. Boeing will deploy a total of 2956 satellites in $1082 \mathrm{~km}, 1030 \mathrm{~km}$ and $970 \mathrm{~km}$ orbits in three phases. At present, most companies are still in the constellation design and networking stage, and there is little research on constellation security. 
In order to further determine the impact of large constellations on the future space debris environment, many scholars have studied large constellations using their own long-term evolution models. References [4-6] studies the impact of the type of constellation disposal orbit, the time in orbit after the mission and the mobility of the satellite on the future environment. References [7-9] have studied the effects of changing the success rate of constellation post-mission disposal (PMD), in-orbit life after mission and the number of satellites in the constellation on the number of space debris in the future. Ref. [10] analyzes the main factors affecting the space debris environment by changing the mass, number, area and PMD orbital height of the launched satellite. Satellites with a larger area and mass are more likely to collide, and more debris are produced after the collision, which has a greater impact on the space environment. The success rate of PMD is an important factor affecting the sustainable development of the space environment, which can directly reflect the growth rate of space debris. Therefore, the performance of the satellite with low mass and a small area is better, the mitigation guidelines should be strictly observed when launching large constellations, and the satellite should have the ability to avoid a collision.

The above studies focus on the impact of individual factors of the number, area and mass of satellites in the constellation on the future environment, but lack of comprehensive analysis of the impact of the number, area and mass of satellites in the constellation on the long-term evolution results. and the impact of different deployment heights of constellations and the interaction between satellites in constellations on the environment is not deeply considered. In this paper, taking the Starlink constellation as an example, the interaction between the constellation and the space debris environment is discussed, and the orbital evolution of the constellation during the mission cycle is analyzed. In different mission stages, the flux analysis of individual satellite and overall satellite constellation is carried out, and calculate the collision risk assessment. According to the different operating heights and inclination of the constellation, the number of avoidance maneuvers and risk levels are evaluated, and the impact of the success rate of post mission disposal (PMD) on the space environment is analyzed. Finally, in the conclusion, the interaction between the space debris environment and the large satellite constellation is given, and the constellation satellite protection and mitigation suggestions are provided.

The structure of the paper is as follows. Section 2 introduces the deployment and situation of the Starlink constellation. Section 3 evaluates the collision risk of satellites and constellations in different mission stages. Section 4 analyzes the avoidance maneuver of the constellation and the corresponding risk level. Section 5 gives the impact of the success rate of constellation PMD. Section 6 gives the discussion and conclusions.

\section{The Starlink Constellation}

According to data published by SpaceX and the Space-track website [11], the Starlink constellation plans to deploy a total of 42,000 satellites in LEO by four phases, as shown in Table 1. Each satellite will have a mass of about $260 \mathrm{~kg}$, the design life is 5 years, and the orbit alttitude is adjusted by electric propulsion. At present, the project has completed the construction of the first phase of the orbital layer, and the number of satellites in orbit has reached 1628.

Table 1. Starlink constellation plan.

\begin{tabular}{cccc}
\hline Phase & Orbital Altitude $\mathbf{( k m )}$ & Inclination $\left.\mathbf{(}^{\circ}\right)$ & Number of Satellites \\
\hline i & 550 & 53.0 & 1584 \\
ii & 540 & 53.2 & 1584 \\
& 570 & 70.0 & 720 \\
& 560 & 97.6 & 348 \\
& 560 & 97.6 & 172 \\
iii & 340 & $42-53$ & 7518 \\
iv & $328-580$ & $30-87.7$ & 30,000 \\
\hline
\end{tabular}


Starlink satellite is released at $300 \mathrm{~km}$ circular orbit, and climb to the mission orbit at $550 \mathrm{~km}$ altitude in three months, then active mission for 5 years. The satellite is equipped with an active collision-avoidance system to reduce the impact of satellite collision. At the end of the mission, the satellite took the initiative to break out of orbit and re-enter the atmosphere to be destroyed. According to the first test satellites launched in May 2019, $95 \%$ of the parts of the satellite during the reentry phase can be burned quickly and do not endanger the operation of other spacecraft.

The mission of the Starlink satellite is split into three phases:

1. Release at $53^{\circ}$ inclination at $300 \mathrm{~km}$, and climb to the mission orbit of $550 \mathrm{~km}$ in 2-3 months.

2. Running normally on the mission orbit for 5 years.

3. Decelerating by an electric engine to re-enter the atmosphere, burn-up and be destroyed.

The Starlink satellite is characterized by a flat plate design with a size of $2.8 \mathrm{~m} \times 1.4 \mathrm{~m}$ $\times 0.15 \mathrm{~m}$, with one solar panel with the length of $9 \mathrm{~m} \mathrm{[12]}$. The maximum cross-section of the satellite is calculated to be $25.2 \mathrm{~m}^{2}$.

\section{Risk Analysis of Constellation Collision}

When describing the risk of collision between the space debris environment and the constellation, it is necessary to evaluate the debris size threshold that can cause catastrophic collisions. The Energy-to-Mass ratio $(E M R)$ is used as a measure of catastrophic collision events, which is defined as

$$
E M R=\frac{M_{i m p} v_{r e l}^{2}}{2 M_{t a r}},
$$

where $M_{\text {imp }}$ describes the mass of the debris, $v_{r e l}$ is the velocity between satellite and target debris, $M_{t a r}$ is the satellite mass. If the $E M R$ exceeds the $40,000 \mathrm{~J} / \mathrm{kg}$, the collision is considered to be catastrophic [13], which means the satellite is completely broken. Through the analysis of the historical collision event, taking the average velocity $10 \mathrm{~km} / \mathrm{s}$, it is calculated that the mass of the target debris in the catastrophic collision is $0.210 \mathrm{~kg}$, assuming that the target debris to be sphere [14], and the density is $2.8 \mathrm{~g} / \mathrm{cm}^{3}$, which is similar to that of the aluminum material. The diameter threshold of the catastrophic collision is about $6 \mathrm{~cm}$, which is smaller than the detectable threshold, $10 \mathrm{~cm}$. Therefore, it is impossible to use the available anti-collision technology to avoid it.

Based on the flux F calculated in the MASTER model [15], determine the average number of collisions of the satellite [16], which is defined as

$$
N=F A T,
$$

where $T$ is the running time range of the satellite, and $A$ is the collision cross-sectional area, which can be calculated according to the radius of the satellite and the target debris

$$
A=\pi\left(r_{t a r}+r_{i m p}\right)^{2} .
$$

According to the average number of collisions, Poisson distribution is used to calculate the probability distribution of $n$ collisions

$$
P_{n}=\frac{N^{n}}{n !} e^{-N}
$$

The probability of no impact $(n=0)$ is

$$
P_{n P}=e^{-N}
$$


The probability of one or more impact is

$$
P=1-P_{n P} .
$$

\subsection{Phase 1: Climbing from $300 \mathrm{~km}$ to $550 \mathrm{~km}$}

After the release of each batch of Starlink satellites, the climbing process and orbital height changes are shown in Figure 1. The first batch lasts about 41 days, the second batch lasts about 65 days, and the third batch lasts about 115 days. The average climbing days of 73 days are selected as the time parameter. During the climbing phase, the evolution of the flux and orbital height of Starlink satellites encountering $\geq 6 \mathrm{~cm}$ objects over time is shown in Figure 2. At this stage, the overall objects flux fluctuates between $2.52 \times 10^{-6} / \mathrm{m}^{2} / \mathrm{a}$ to $2.59 \times 10^{-6} / \mathrm{m}^{2} / \mathrm{a}$, and the highest flux is about $2.59 \times 10^{-6} / \mathrm{m}^{2} / \mathrm{a}$ during the adjustment waiting for orbit ascent.

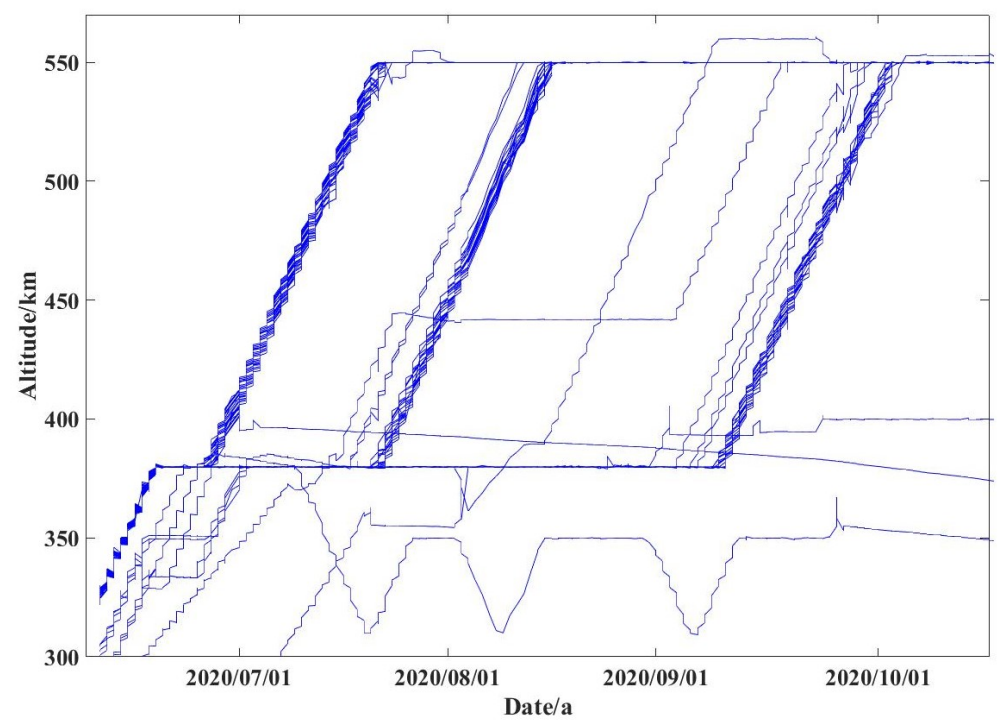

Figure 1. Starlink satellite orbit altitude.

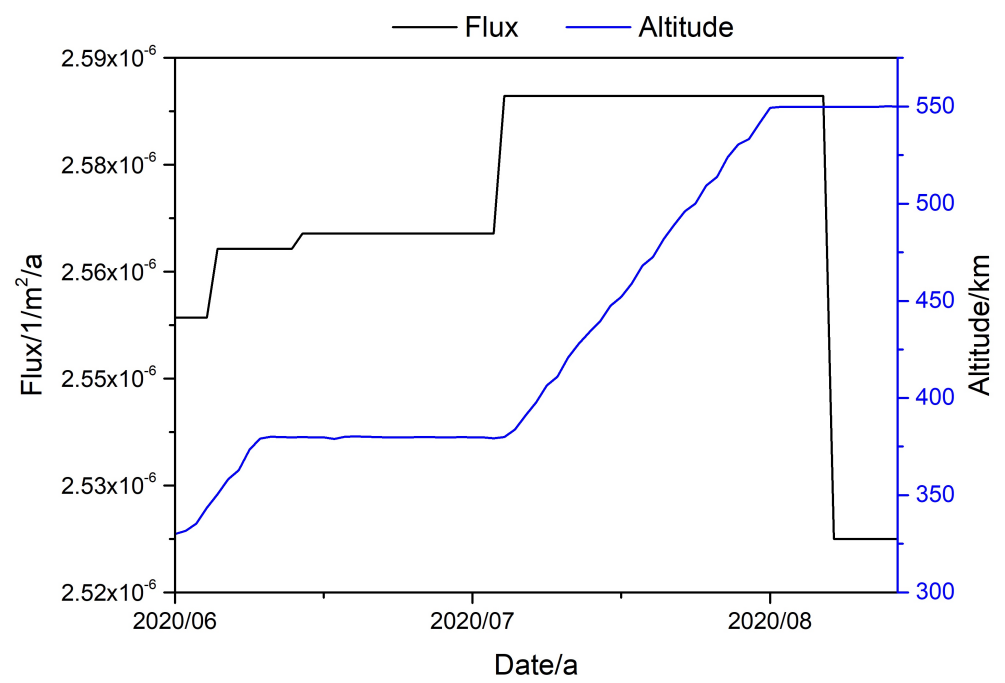

Figure 2. Flux and satellites altitude over time during Phase 1 with objects $\geq 6 \mathrm{~cm}$.

The average number of collisions and the relationship between the collision probability and the debris diameter of a single satellite and all satellites in the orbital layer during the climbing phase are shown in Table 2. The collision probability of debris larger than $6 \mathrm{~cm}$ 
to a single satellite is less than $0.01 \%$, and the collision probability of 1628 satellites in the orbital layer is $1.89 \%$.

Table 2. Fluxes, numbers of impacts and probability for one or more collisions for constellation objects during Phase 1.

\begin{tabular}{cccccc}
\hline Class & Flux (1/m $\left.\mathbf{m}^{2} / \mathbf{a}\right)$ & $\mathbf{N}$ & $\boldsymbol{P}_{\geq \mathbf{1}}(\mathbf{\%})$ & $\boldsymbol{N}_{\mathbf{1 6 2 8}}$ & $\boldsymbol{P}_{\geq \mathbf{1 , 1 6 2 8}}(\%)$ \\
\hline$d \geq 1 \mathrm{~cm}$ & $3.16 \times 10^{-5}$ & $1.42 \times 10^{-4}$ & 0.02 & $2.31 \times 10^{-1}$ & 20.60 \\
$d \geq 6 \mathrm{~cm}$ & $2.56 \times 10^{-6}$ & $1.17 \times 10^{-5}$ & $<0.01$ & $1.91 \times 10^{-2}$ & 1.89 \\
$d \geq 10 \mathrm{~cm}$ & $1.60 \times 10^{-6}$ & $7.43 \times 10^{-6}$ & $<0.01$ & $1.21 \times 10^{-2}$ & 1.20 \\
$d \geq 1 \mathrm{~m}$ & $5.24 \times 10^{-7}$ & $3.30 \times 10^{-6}$ & $<0.01$ & $5.37 \times 10^{-3}$ & 0.54 \\
\hline
\end{tabular}

\subsection{Phase 2: Operational Time at $550 \mathrm{~km}$}

After reaching the mission orbit, the satellite will operate stably at $550 \mathrm{~km}$ altitude for five years. The time-dependent variation of the flux of $\geq 6 \mathrm{~cm}$ objects encountered by Starlink satellites is shown in Figure 3. Due to the constant height of the satellite, the debris flux fluctuates from $4.50 \times 10^{-6} / \mathrm{m}^{2} /$ a to $6.50 \times 10^{-6} / \mathrm{m}^{2} / \mathrm{a}$, and the overall number has a downward trend.

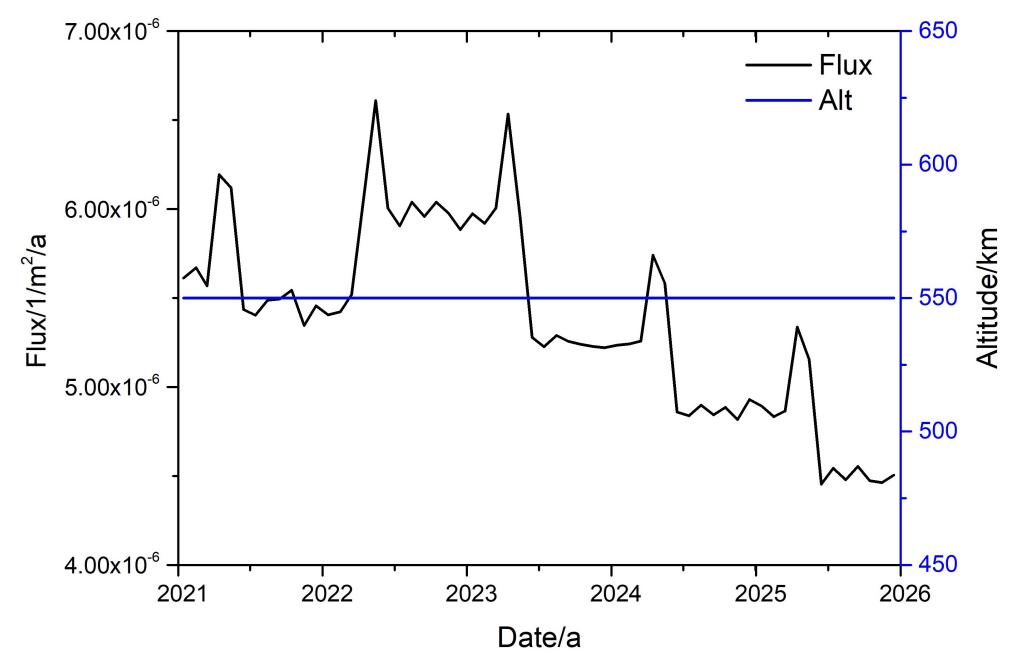

Figure 3. Flux and satellites altitude over time during Phase 2 with objects $\geq 6 \mathrm{~cm}$.

The average number of collisions and the relationship between the collision probability and the debris diameter of a single satellite and all satellites in the orbital layer during the working phase are shown in Table 3. During the 5-year operation, the collision probability of orbiting satellites with objects larger than $1 \mathrm{~cm}$ is $99.99 \%$, and for objects larger than $6 \mathrm{~cm}$ is $63.36 \%$.

Table 3. Fluxes, numbers of impacts and probability for one or more collisions for constellation objects during Phase 2 .

\begin{tabular}{cccccc}
\hline Class & Flux (1/m $\mathbf{m} / \mathbf{a})$ & $\mathbf{N}$ & $\boldsymbol{P}_{\geq \mathbf{1}} \mathbf{( \% )}$ & $\boldsymbol{N}_{\mathbf{1 6 2 8}}$ & $\boldsymbol{P}_{\geq \mathbf{1 , 1 6 2 8}} \mathbf{( \% )}$ \\
\hline$d \geq 1 \mathrm{~cm}$ & $6.58 \times 10^{-5}$ & $8.39 \times 10^{-3}$ & 0.84 & $4.60 \times 10^{-1}$ & 99.99 \\
$d \geq 6 \mathrm{~cm}$ & $4.74 \times 10^{-6}$ & $6.17 \times 10^{-4}$ & 0.06 & $4.19 \times 10^{-2}$ & 63.36 \\
$d \geq 10 \mathrm{~cm}$ & $4.03 \times 10^{-6}$ & $5.30 \times 10^{-4}$ & 0.05 & $3.19 \times 10^{-2}$ & 57.80 \\
$d \geq 1 \mathrm{~m}$ & $6.74 \times 10^{-7}$ & $1.18 \times 10^{-4}$ & 0.01 & $7.71 \times 10^{-3}$ & 17.53 \\
\hline
\end{tabular}

\subsection{Phase 3: Take Derailment Disposal, Decelerate and Deviate from the Mission Orbit}

The power system of the Starlink satellite uses a krypton ion thruster, which triggers the active derailment device after the five-year operation mission, and the thruster con- 
tinues to apply small thrust to reduce the satellite's orbital altitude, and finally, enter the atmosphere to burn and destroy. The duration of this stage is about 9.7 months. The variation of the flux of the satellite encountering $\geq 6 \mathrm{~cm}$ objects with time and the evolution of the satellite orbital height are shown in Figure 4. At the beginning of satellite disposal, the flux of the object fluctuates from $2.03 \times 10^{-6} / \mathrm{m}^{2} / \mathrm{a}$ to $2.21 \times 10^{-6} / \mathrm{m}^{2} / \mathrm{a}$, the satellite orbit altitude reaches $350 \mathrm{~km}$ is affected by atmospheric resistance. The orbit height decreases rapidly and enters the atmosphere for combustion and destruction.

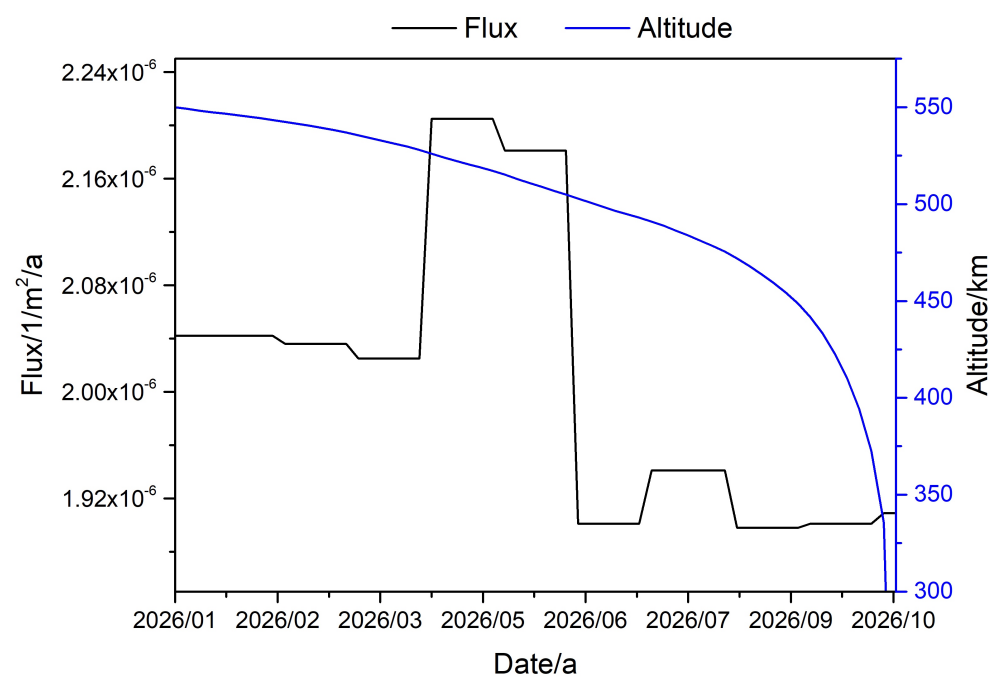

Figure 4. Flux and satellites altitude over time during Phase 3 with objects $\geq 6 \mathrm{~cm}$.

The relationship between the average collision times, collision probability and objects diameter of a single satellite and all satellites in the orbital layer during the out-of-orbit disposal phase is shown in Table 4. In the out-of-orbit disposal phase, the collision probability of the orbital layer satellite with objects larger than $1 \mathrm{~cm}$ is $50.15 \%$, and the collision probability with objects larger than $6 \mathrm{~cm}$ is $6.83 \%$.

Table 4. Fluxes, numbers of impacts and probability for one or more collisions for constellation objects during Phase 3 .

\begin{tabular}{|c|c|c|c|c|c|}
\hline Class & Flux $\left(1 / \mathrm{m}^{2} / a\right)$ & $\mathbf{N}$ & $P_{\geq 1}(\%)$ & $N_{1628}$ & $P_{\geq 1,1628}(\%)$ \\
\hline$d \geq 1 \mathrm{~cm}$ & $2.21 \times 10^{-5}$ & $4.28 \times 10^{-4}$ & 0.04 & $6.96 \times 10^{-1}$ & 50.15 \\
\hline$d \geq 6 \mathrm{~cm}$ & $2.20 \times 10^{-6}$ & $4.34 \times 10^{-5}$ & 0.01 & $7.07 \times 10^{-2}$ & 6.83 \\
\hline$d \geq 10 \mathrm{~cm}$ & $1.37 \times 10^{-6}$ & $2.73 \times 10^{-5}$ & $<0.01$ & $4.45 \times 10^{-2}$ & 4.35 \\
\hline$d \geq 1 \mathrm{~m}$ & $5.35 \times 10^{-7}$ & $1.44 \times 10^{-5}$ & $<0.01$ & $2.34 \times 10^{-2}$ & 2.32 \\
\hline
\end{tabular}

\subsection{Starlnik Constellation Collision Risk}

SpaceX has given the planning parameters of about 38,000 satellites expected to be deployed in Phase iii and iv, but the final deployment mode still needs to be adjusted according to the actual use after the completion of the networking of Phase $i$ and Phase ii. Therefore, in this paper, only the Phase i satellites have already been in orbit and the Phase ii satellites to be launched are used in the overall analysis of the Starlink constellation, with a total of 4452 satellites. It can be seen from Table 1 that phase ii satellites are deployed in four orbital layers. The height of the first orbital layer is $540 \mathrm{~km}$ and the inclination is $53.2^{\circ}$. There are 1584 satellites in total. The height of the second orbital layer is $570 \mathrm{~km}$ and the inclination is $70^{\circ}$ with 720 satellites. The main orbital parameters of the third orbital layer and the fourth orbital layer are the same, the altitude is $560 \mathrm{~km}$, the inclination is $97.6^{\circ}$, a total of 520 satellites. Similarly, the complete mission cycle of the satellite is divided into three stages to evaluate the space object flux and collision risk in the orbit of a single 
satellite, and to calculate the overall collision probability of the satellite in the orbital layer. The flux variation of the objects above $6 \mathrm{~cm}$ encountered by satellites with different orbital layers in the Starlink constellation during the mission cycle is shown in Figure 5, and the single and overall collision probabilities of satellites with different orbital layers are shown in Table 5.

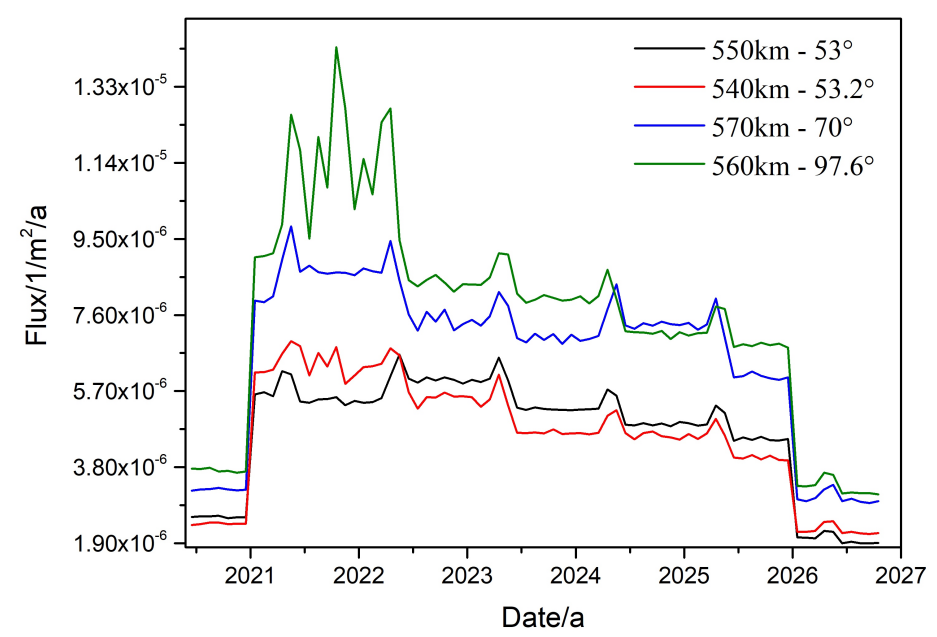

Figure 5. Flux of objects on satellites with different orbital parameters with objects $\geq 6 \mathrm{~cm}$.

Table 5. Probability of collision between satellites in different orbital layers and objects $\geq 6 \mathrm{~cm}$.

\begin{tabular}{|c|c|c|c|c|c|c|c|c|}
\hline \multirow[t]{2}{*}{ Phase } & \multicolumn{4}{|c|}{ Single Satellite } & \multicolumn{4}{|c|}{ All Satellites } \\
\hline & $\begin{array}{l}P_{53^{\circ}} \\
(\%)\end{array}$ & $\begin{array}{c}P_{53.2^{\circ}} \\
(\%)\end{array}$ & $\begin{array}{l}P_{70^{\circ}} \\
(\%)\end{array}$ & $\begin{array}{c}P_{97.6^{\circ}} \\
(\%)\end{array}$ & $\begin{array}{l}P_{53^{\circ}} \\
(\%)\end{array}$ & $\begin{array}{c}P_{53.2^{\circ}} \\
(\%)\end{array}$ & $\begin{array}{l}P_{70^{\circ}} \\
(\%)\end{array}$ & $\begin{array}{c}P_{97.6^{\circ}} \\
(\%)\end{array}$ \\
\hline 1 & $<0.01$ & $<0.01$ & $<0.01$ & $<0.01$ & 1.89 & 1.72 & 1.06 & 0.88 \\
\hline 2 & 0.06 & 0.07 & 0.10 & 0.12 & 63.36 & 66.25 & 50.88 & 44.49 \\
\hline 3 & 0.01 & 0.01 & 0.01 & 0.01 & 6.83 & 6.69 & 4.21 & 3.32 \\
\hline
\end{tabular}

In the working stage of the satellite, when satellites have a similar orbit, the object flux in the orbit with a $97.6^{\circ}$ inclination is about twice as much as that at the inclination of $53^{\circ}$, and the collision risk of the satellite in the orbit with high inclination is higher. When satellites have a similar inclination, the initial flux of the space object at the $540 \mathrm{~km}$ is slightly larger than that of the $550 \mathrm{~km}$, but due to the influence of atmospheric resistance, the fading speed of the object with low orbit is faster, and the average flux at the $550 \mathrm{~km}$ is larger during the overall working time in orbit, so the risk of satellite collision is higher. As can be seen from Table 5, the collision probability of a single satellite increases with the promotion of the orbital inclination, but because of more satellites in the orbital layer near the low inclination, the corresponding overall collision probability is higher. In the whole mission cycle of the satellite life, the collision risk is the highest in the in-orbit operation phase, so we need to pay more attention to the design of collision avoidance and in-orbit satellite monitoring.

\section{Analysis of Constellation Collision Maneuver}

Starlink satellites can take evasive maneuver measures on the basis of collision early warning based on the cataloged object orbit data provided by the US Space Surveillance Network, but because the thrust provided by ion thruster is small, the fuel or working medium that the satellite can carry is limited, and the operation time of each avoidance maneuver is longer than several minutes. A too-high maneuvering frequency or long-term altitude changes will lead to a constellation configuration change. Therefore, it is necessary to selectively avoid possible collisions. When formulating the avoidance strategy in the design stage of the satellite, the accepted collision probability level (ACPL) is usually 
used as the evaluation criterion to measure whether to take evasive measures or not. For satellites operating in LEO orbits, the ACPL values used in the design are between $10^{-4}$ and $10^{-5}$ [17].

The DRAMA software [18] developed by the ESA is used to analyze the avoidance maneuver times and collision risk of 1628 Starlink satellites deployed in phase $i$. The cross-section of the satellite is $25.2 \mathrm{~m}^{2}$, the equivalent radius is $2.667 \mathrm{~m}$, and the collision risk of objects of $6 \mathrm{~cm}$ and above is considered.

\subsection{Phase 1: Collision Avoidance Maneuver during Climbing}

In order to simplify the calculation process of the model, the satellite orbit in the climbing phase is approximately an elliptical orbit of $300 \mathrm{~km} \times 550 \mathrm{~km}$, the semi-major axis is $6803 \mathrm{~km}$, the eccentricity is 0.0184 and the orbit inclination is $53^{\circ}$. The calculation time is 73 days. The calculated results are described by two data, one is detectable, that is, the collision probability and flux of objects above $10 \mathrm{~cm}$ (subscript $\mathrm{d}$ ), and the other is the overall collision probability and flux of space objects, including undetectable objects evolved from the space debris model (subscript w), as shown in Table 6.

Table 6. Single satellite annual collision probability (ACP) and space object flux during Phase 1.

\begin{tabular}{cccc}
\hline$A C P_{d}$ & $\boldsymbol{A C P}_{w}$ & Flux $_{\boldsymbol{d}}\left(/ \mathrm{m}^{2} / \mathbf{a}\right)$ & Flux $_{w}\left(/ \mathrm{m}^{2} / \mathbf{a}\right)$ \\
\hline $8.22 \times 10^{-5}$ & $8.51 \times 10^{-5}$ & $2.42 \times 10^{-6}$ & $2.56 \times 10^{-6}$ \\
\hline
\end{tabular}

The ACPL value is defined by the engineer as a parameter that is beneficial to the efficient avoidance and maneuver of the satellite. The value range of ACPL in actual operation is comprehensively considered by calculating the avoidance times of the satellite under a certain collision probability level. It can be seen from the data in Table 5 that, although the collision probability of a single satellite at the inclination of the high orbit is larger, the overall collision probability of the orbital layer is smaller due to the influence of the number of satellites. Therefore, when calculating the avoidance times of all satellites in a constellation corresponding to different ACPL values, it is necessary to optimize the average avoidance times of the single satellites in this environment, so as to obtain the avoidance maneuver times corresponding to different ACPL values in this orbital layer.

Figure 6 shows the variation of the total avoidance times of 1628 Starlink satellites with the ACPL value during the climbing phase. when the ACPL value is $10^{-6}$, the satellite in this orbital layer needs about 2000 maneuvers to avoid, and a low ACPL value will reduce the operation and efficiency of the satellite. When the ACPL value is $10^{-4}$, the orbital layer satellite only needs to avoid three times. Figure 7 shows the relationship between the overall collision risk and the number of avoidance. The total collision risk is reduced by $0.2 \%$ (Risk Reduction) due to three avoidance maneuvers, and the residual risk of detectable objects and remaining risk of all objects is about $1.5 \%$, indicating that the impact of non-detectable objects with diameters between 6-10 cm on satellite collision risk is small. 


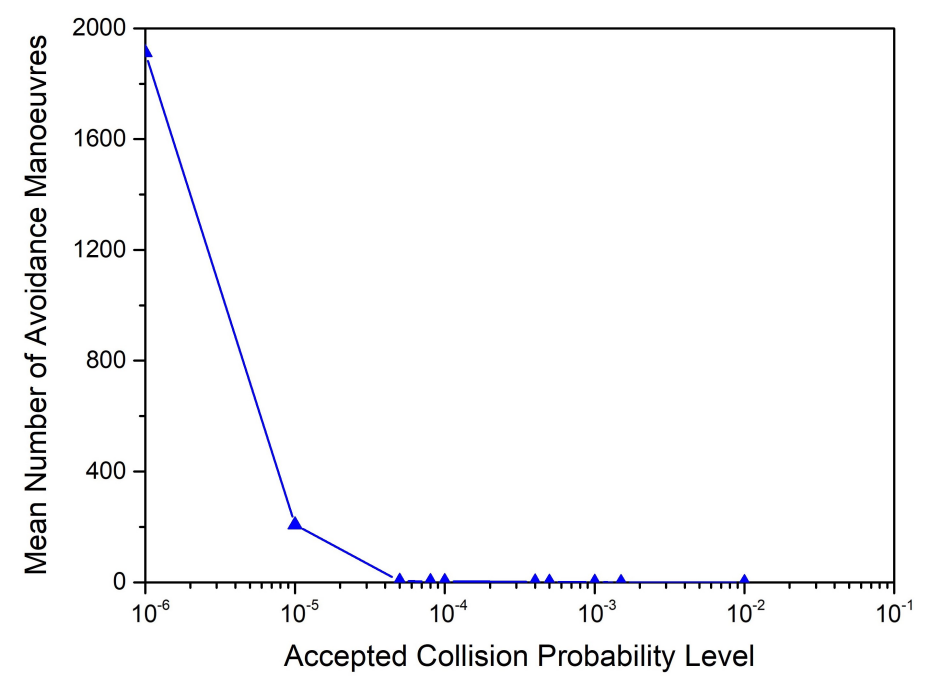

Figure 6. The relationship between the mean number of avoidance manoeuvres and ACPL during Phase 1.

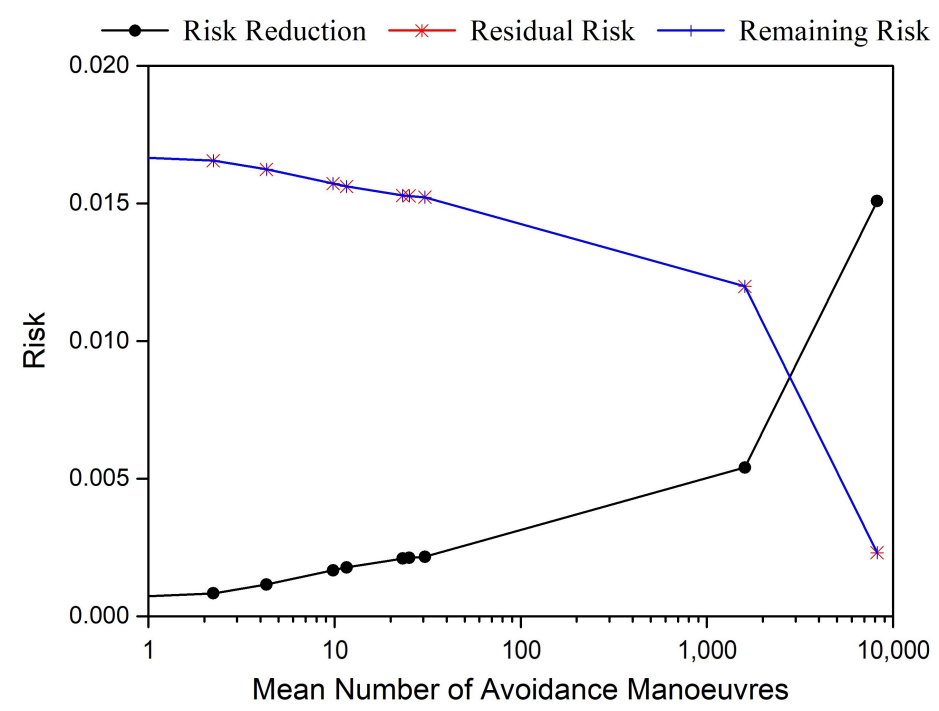

Figure 7. The relationship between risk and the mean number of avoidance manoeuvres during Phase 1.

\subsection{Phase 2: Collision Avoidance Maneuver during Working in Orbit}

During the operation of the satellite, it operates in a near-circular orbit at the height of $550 \mathrm{~km}$, with a semi-major axis of $6928 \mathrm{~km}$, an orbital inclination of $53^{\circ}$ and an eccentricity of 0.0001 . The calculation time is from January 2021. The on-orbit collision avoidance simulation is carried out for 5 years, and the annual collision probability and flux with detectable objects and all objects are obtained, as shown in Table 7.

Table 7. Single satellite annual collision probability (ACP) and space object flux during Phase 2.

\begin{tabular}{cccc}
\hline$A C P_{d}$ & $A C P_{w}$ & Flux $_{d}\left(/ \mathrm{m}^{2} / \mathbf{a}\right)$ & Flux $_{w}\left(/ \mathbf{m}^{2} / \mathbf{a}\right)$ \\
\hline $1.67 \times 10^{-4}$ & $1.72 \times 10^{-4}$ & $4.58 \times 10^{-6}$ & $4.74 \times 10^{-6}$ \\
\hline
\end{tabular}

Figure 8 shows the relationship between the total number of circumvention and ACPL value during satellite operation in orbit. When the ACPL value is $10^{-4}$, the total number of avoidance of all satellites is about 1300 , with an average annual avoidance of 260 , as can be seen from Figure 9, if the corresponding full maneuver avoidance is carried out, the 
collision risk can be reduced by $60 \%$, and the remaining collision risk for all space objects is $20 \%$. When the ACPL value is $10^{-5}$, all mobile avoidance can reduce the remaining risk to less than $5 \%$, but too many avoidance times will lead to an increase in fuel demand, so it is necessary to make a reasonable choice of ACPL value according to the mission situation.

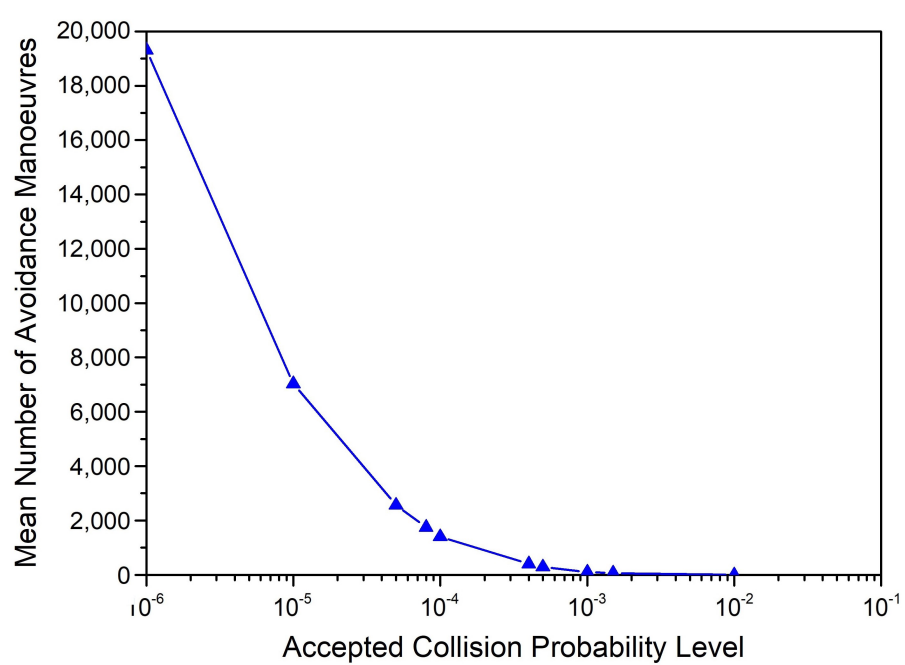

Figure 8. The relationship between the mean number of avoidance manoeuvres and ACPL during Phase 2.

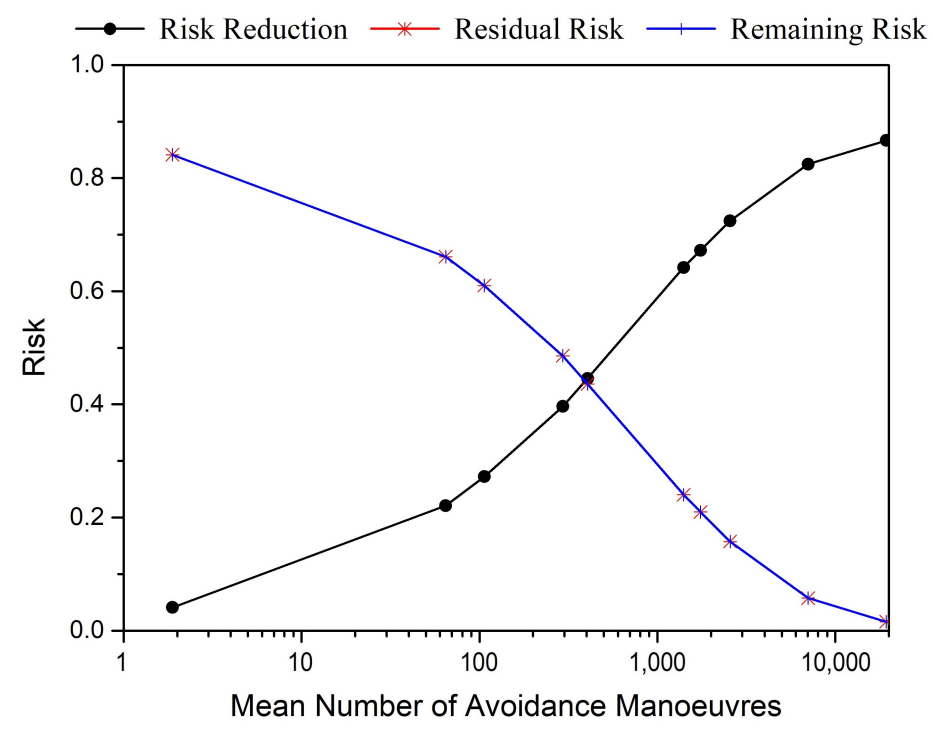

Figure 9. The relationship between risk and the mean number of avoidance manoeuvres during Phase 2.

\subsection{Phase 3: Collision Avoidance Maneuver in the Stage of Derailment Disposal}

As can be seen from Figure 4, the fading speed accelerates when the satellite orbit altitude is lower than that of $350 \mathrm{~km}$, and the debris is highly affected by the atmosphere and the space object flux is low, and the collision between the two is relatively small. The orbit of the satellite in the out-of-orbit phase is approximate to the elliptical orbit of $550 \mathrm{~km} \times 350 \mathrm{~km}$, the semi-major axis is $6828 \mathrm{~km}$, the eccentricity is 0.01465 , the inclination of the orbit is $53^{\circ}$, and the calculation time is 293 days. the annual collision probabilities and fluxes of detectable objects and all objects are obtained as shown in Table 8. 
Table 8. Single satellite annual collision probability (ACP) and space object flux during Phase 3.

\begin{tabular}{cccc}
\hline$A C P_{d}$ & $A C P_{w}$ & Flux $_{d}\left(/ \mathbf{m}^{2} / \mathbf{a}\right)$ & Flux $_{w}\left(/ \mathbf{m}^{2} / \mathbf{a}\right)$ \\
\hline $7.76 \times 10^{-5}$ & $7.78 \times 10^{-5}$ & $2.20 \times 10^{-6}$ & $2.20 \times 10^{-6}$ \\
\hline
\end{tabular}

Figure 10 shows the relationship between the overall avoidance times and the ACPL value of the satellite in the out-of-orbit disposal phase. When the ACPL value is $10^{-4}$, all satellites need to perform 15 avoidance maneuvers at this stage. As shown in Figure 11, the risk after the maneuver is reduced by $1 \%$, and the collision remaining risk caused by all space objects is $5.7 \%$. By increasing the number of avoidances, the remaining risk can continue to be reduced.

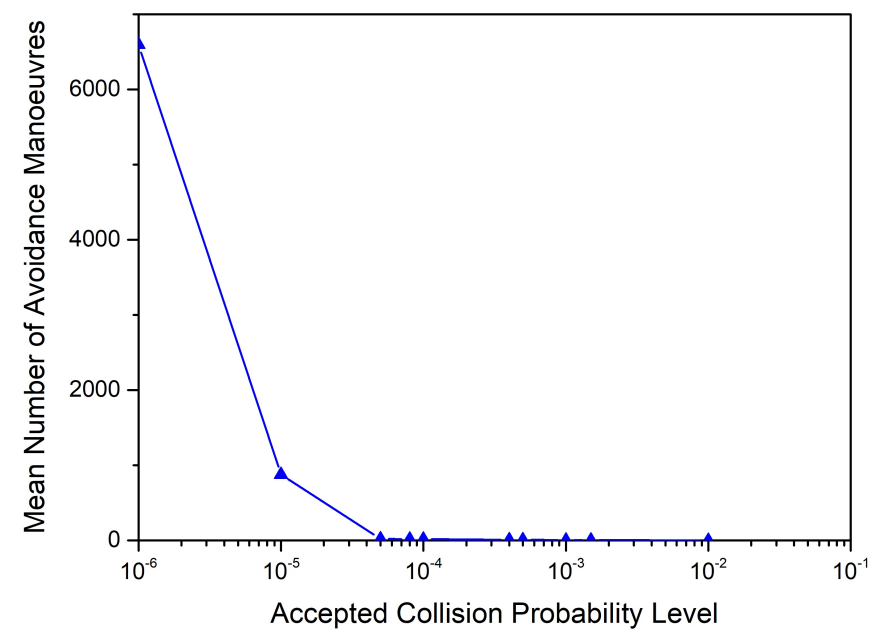

Figure 10. The relationship between the mean number of avoidance manoeuvres and ACPL during Phase 3.

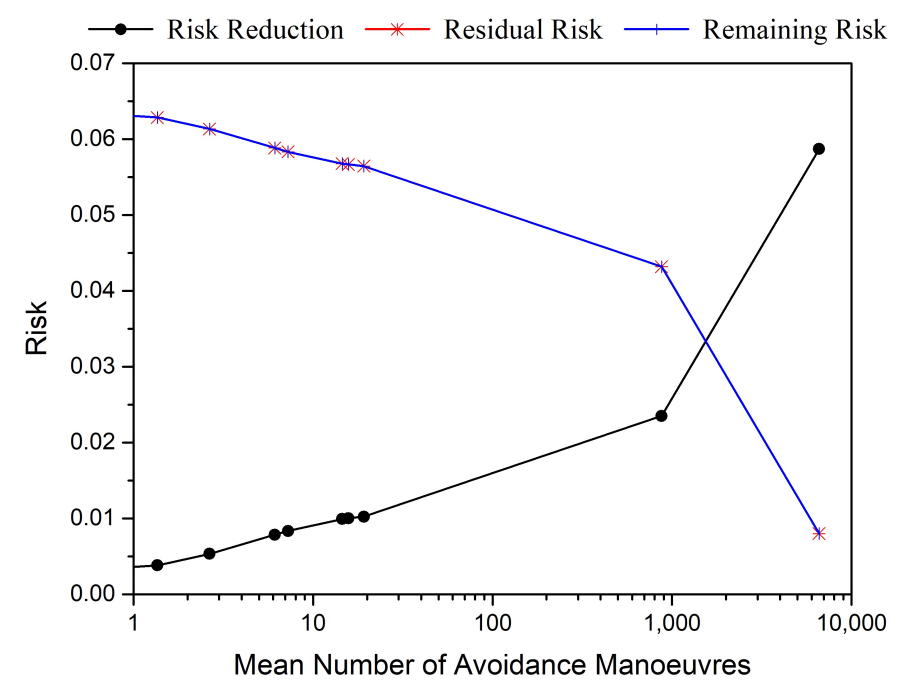

Figure 11. The relationship between risk and the mean number of avoidance manoeuvres during Phase 3.

\subsection{Starlink Constellation Collision Avoidance Maneuver}

Based on the analysis of the collision avoidance maneuvers of the other three orbital layers mentioned in Section 3.4, Table 9 shows the average collision probability and average object flux of a single satellite in each orbital layer during the mission. When the value of ACPL is $10^{-4}$, the total avoidance maneuver times of the satellite in each stage and in the whole mission under different orbit parameters are shown in Figure 12. The flux 
of space objects in the orbit with high inclination is larger, and more collision avoidance maneuvers are needed in the climbing phase to ensure the safe and orderly follow-up work of the satellite.

When the orbit height is similar, the number of avoiding maneuvers in the low inclination orbital layer is about 1500 times during the mission cycle, and the total avoidance times of the high inclination orbital layer satellites is about 500. The satellites on the low inclination orbital layer need to pay more attention to their operation status and replace damaged or failed satellites in time.

Table 9. Average collision probability and average object flux of a single satellite in each orbital layer.

\begin{tabular}{ccccc}
\hline & $\boldsymbol{A C P}_{\boldsymbol{d}}$ & $\boldsymbol{A C P}_{\boldsymbol{w}}$ & $\boldsymbol{F l u x}_{\boldsymbol{d}}\left(/ \mathbf{m}^{2} / \mathbf{a}\right)$ & $\boldsymbol{F l u x}_{w}\left(/ \mathbf{m}^{2} / \mathbf{a}\right)$ \\
\hline $540 \mathrm{~km} / 53.2^{\circ}$ & $1.57 \times 10^{-4}$ & $1.58 \times 10^{-4}$ & $5.37 \times 10^{-6}$ & $5.41 \times 10^{-6}$ \\
$560 \mathrm{~km} / 97.6^{\circ}$ & $2.39 \times 10^{-4}$ & $2.39 \times 10^{-4}$ & $8.39 \times 10^{-6}$ & $8.40 \times 10^{-6}$ \\
$570 \mathrm{~km} / 70^{\circ}$ & $2.15 \times 10^{-4}$ & $2.15 \times 10^{-4}$ & $6.91 \times 10^{-6}$ & $6.91 \times 10^{-6}$ \\
\hline
\end{tabular}

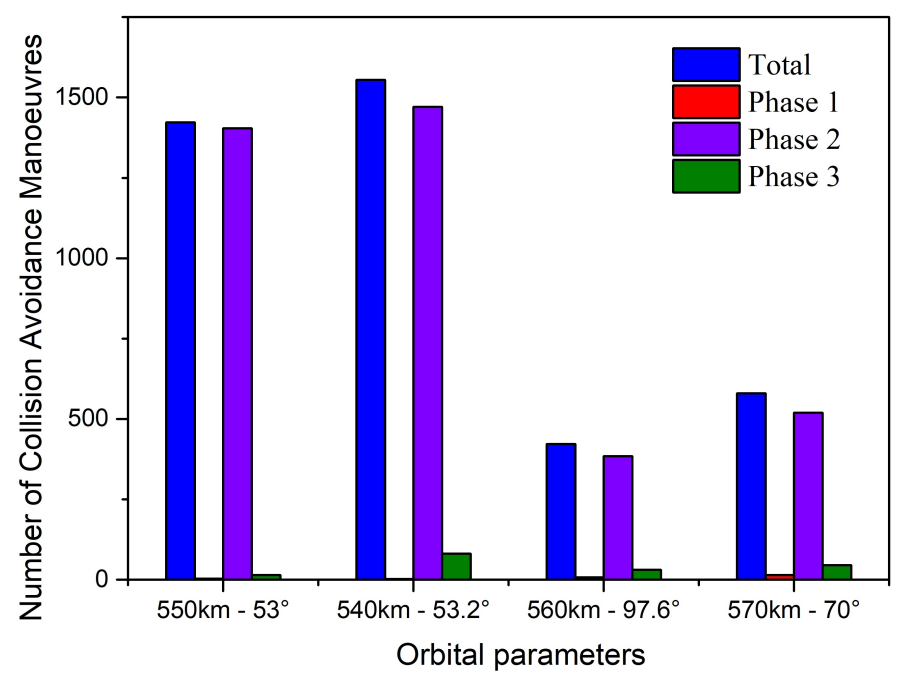

Figure 12. The mean number of avoidance manoeuvres for each orbital satellite.

\section{The Influence of Constellation on Space Environment}

The launch of a large number of constellations will significantly increase the number of space objects, increase the risk of collision between satellites, and have an irreversible impact on the space environment. According to the Delta model [19] developed by the ESA to simulate the evolution of future space objects, considering the effects of the explosion, collision, disintegration and other events as the main sources of debris, it is calculated that the introduced constellation consists of 4452 satellites deployed in phase $i$ and ii of the Starlink constellation. The design life of a single satellite is 5 years, it was first launched in May 2019, and the deployment of all constellations will be complete in 2026. Using the Monte Carlo method $[4,20]$ to calculate the number of space objects above $10 \mathrm{~cm}$ under different conditions in the next 20 years.

\subsection{Impact of Single Satellite Collision}

A large number of debris will be produced after the collision and break up of the satellite in the course of operation. There are two kinds of collision situations: one is the collision between the satellite and the small debris, and the other is the collision between the satellite and the satellite. The parameters such as mass, area-mass ratio and velocity increment produced by the disintegration of space objects can be simulated by using NASA's EVOLVE 4.0 break up model [21], and the initial motion state of the breakup debris cloud can be obtained. This analysis only aims at the collision between the Starlink satellite and other mission satellites in the $550 \mathrm{~km}$ orbit, and the two satellites completely break up 
after the collision. The distribution of fragments larger than $10 \mathrm{~cm}$ is simulated according to the breakup model, as shown in Figures 13 and 14.

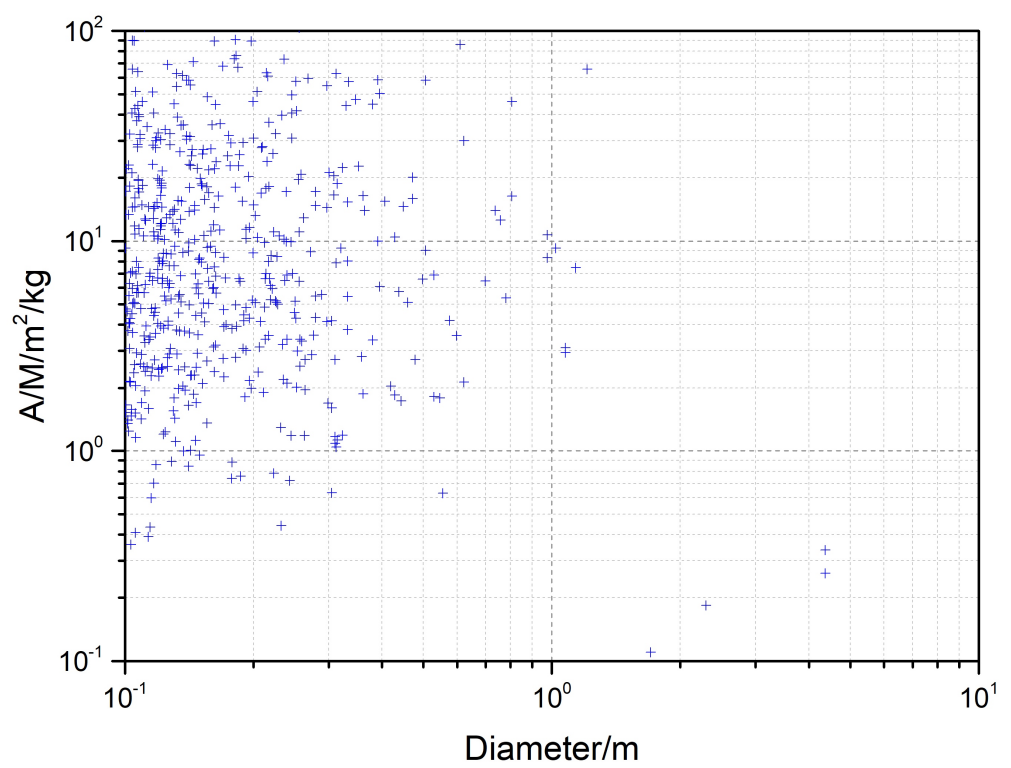

Figure 13. Breakup fragments' area-mass ratio distribution.

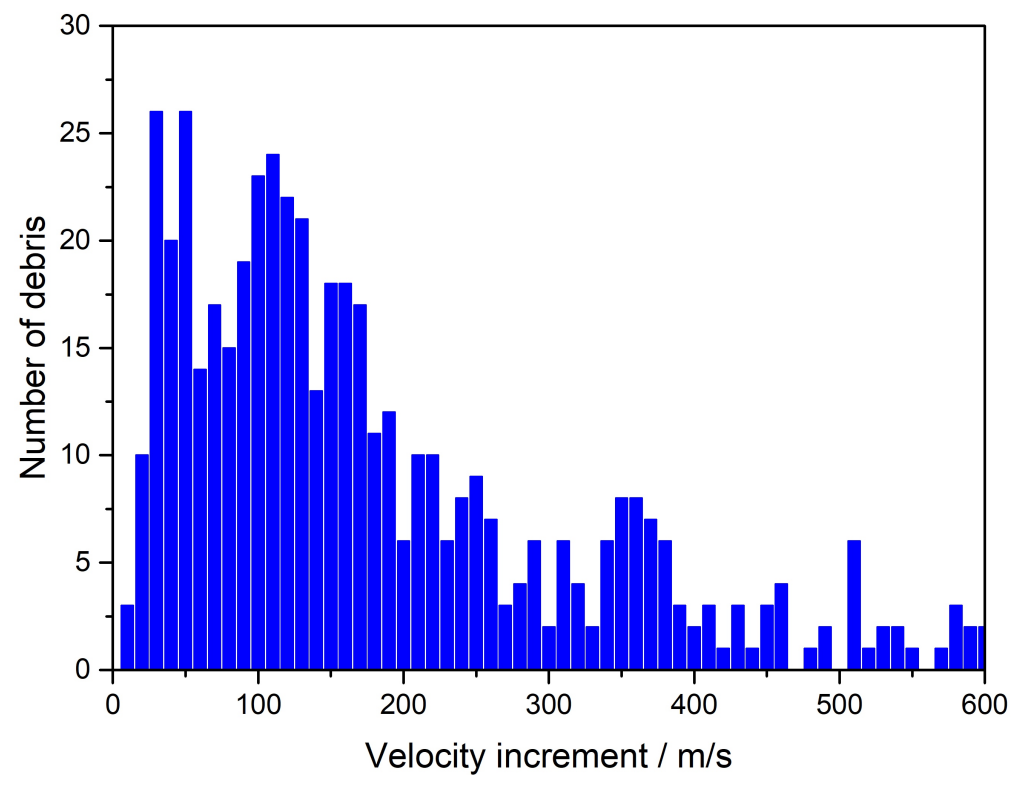

Figure 14. Breakup fragments' velocity increment distribution.

The area-mass ratio can reflect the relationship between the size and quality of debris produced by satellite break up. Through simulation, it is calculated that the number of debris larger than $10 \mathrm{~cm}$ after the collision between Starlink satellites and other mission satellites is about 540 , and the velocity increment of debris is concentrated in 10-150 m/s. Figure 15 shows the orbital distribution larger than that of $10 \mathrm{~cm}$ debris after the collision. Because the orbit of the satellite is a $550 \mathrm{~km}$ circle orbit, the orbit height of the breakup debris is mainly concentrated in this area, which has a great impact on the parent satellite and there is a collision threat. 


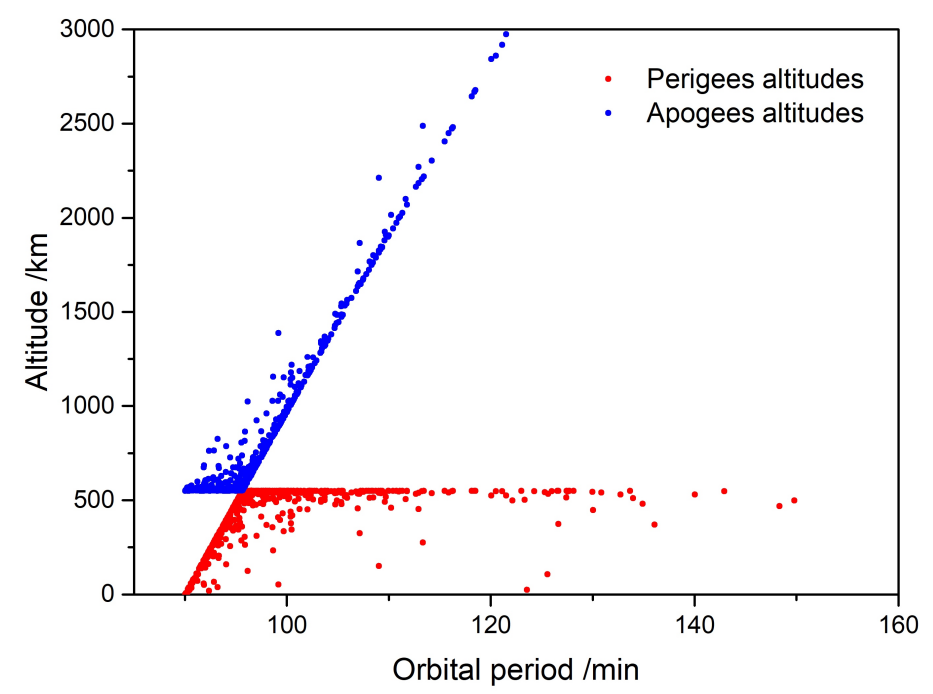

Figure 15. Breakup fragments' orbit distribution.

\subsection{Objects Number Influenced by Constellation}

Based on the collision simulation of a single satellite, the collision influence of the constellation is analyzed comprehensively. Figure 16 shows the evolution of the number of space objects larger or equal to $10 \mathrm{~cm}$ with and without constellations. After joining constellations, the number of objects increases obviously at the beginning and then fluctuates. The blue line represents the proportion of space objects with and without constellations, which is on the rise, indicating that the existence of constellations has accelerated the growth of the number of objects, and by 2040, the number of space objects will be nearly 1.3 times higher than when there are none constellations. This is only the impact of some satellites in the Starlink constellation plan. In the later stage, after all 38,000 satellites are deployed, the impact on the number of objects will increase exponentially, and the collision risk will increase step by step. The high requirements for configuration and accuracy of constellation satellites may even lead to cascade collisions.

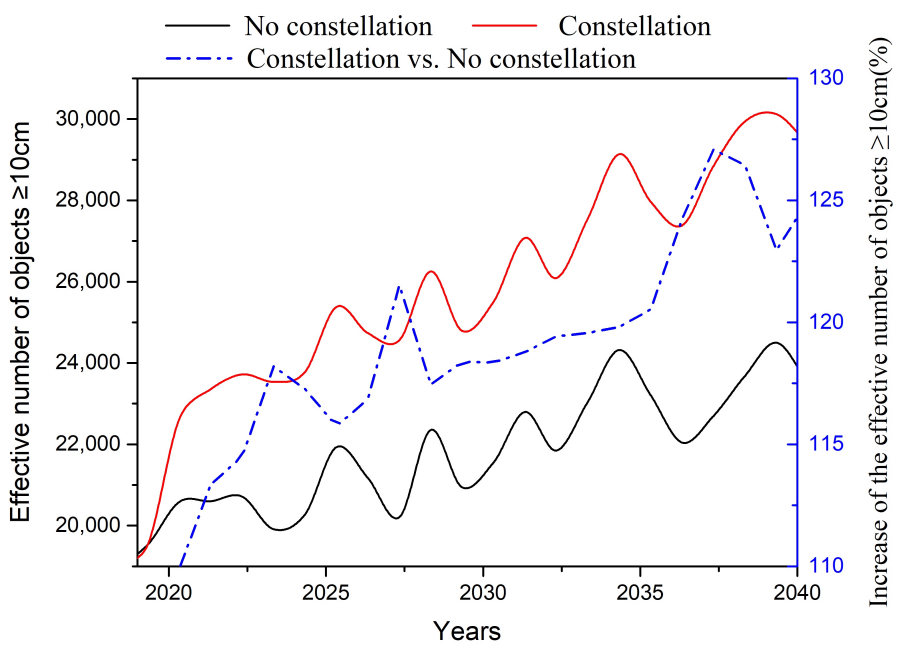

Figure 16. The influence of constellations on the evolution of objects more than $10 \mathrm{~cm}$.

\subsection{PMD Rate Influence}

Although the Starlink satellite adds the post-mission active derailment link in the design phase, if the satellite collides or is damaged during the mission cycle, the active derailment device can not be effectively triggered, resulting in a longer period of satellite re-entry into the atmosphere and increasing the impact on the space environment, so it is necessary to analyze the post-mission disposal (PMD) success rate. 
Figure 17 compares the object growth trend under the four PMD success rates of $50 \%, 75 \%, 90 \%, 100 \%$. The increase in the number of objects corresponding to the higher PMD rate at the initial stage of deployment is more moderate. After the completion of deployment, the growth trend of objects corresponding to the four PMD rates is similar, and the growth rate of objects with $90 \%$ and $100 \%$ PMD success rates slows down in the later stage. The lower the PMD rate, the higher the number of satellites left in orbit, the greater the probability of collision with the passage of time, and the interaction between the accumulation of satellites and space objects will lead to the continuous deterioration of the space environment.

Figure 18 shows the ratio of the number of objects corresponding to different PMD rates to the number of objects without constellations. The higher PMD rate can reduce the growth rate of space objects, but due to the influence of constellations, the growth rate of objects continues to rise. By 2040, even with the PMD success rate up to $100 \%$, the growth rate of objects will still be about 1.07. Therefore, the introduction of large satellite constellations is bound to have an impact on the space environment and the growth of space objects, and the risk of this impact can be reduced to a certain extent by improving the success rate of PMD.

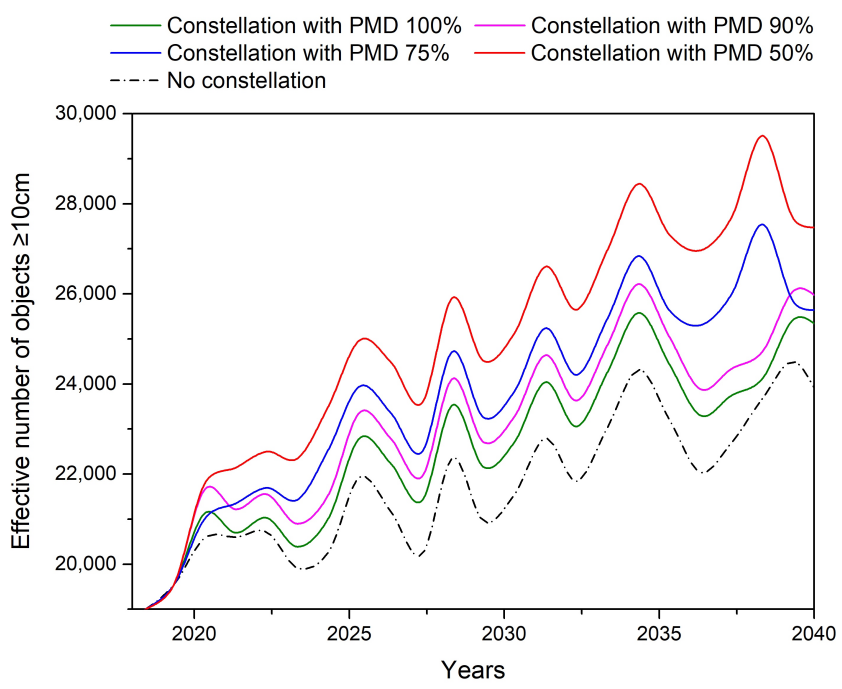

Figure 17. The influence of different PMD success rates on the evolution of objects.

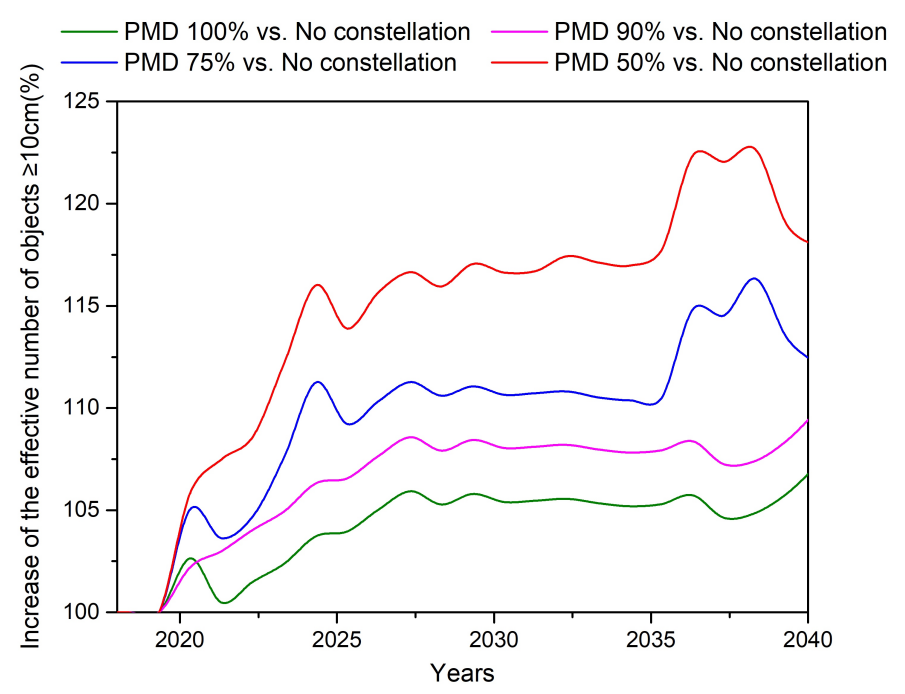

Figure 18. The ratio of different PMD success rates and objects without joining constellations. 


\section{Discussion}

In this paper, the Starlink constellation planned by SpaceX Company is used as the research object. By changing the number of satellites in the constellation, the orbital height and inclination of the constellation, the effects of the launch of large constellations on space object flux, collision probability, avoidance maneuver times and future space debris environments are analyzed.

According to the calculation, objects above $6 \mathrm{~cm}$ will cause catastrophic collisions of Starlink satellites, and the maximum flux on the satellite is during the working period in orbit, and the long mission cycle further increases the collision risk of this stage. In addition, the collision of the three orbital layers planned to be launched by the Starlink constellation is also analyzed. when the orbital height is similar, the collision probability of a single satellite increases with the promotion of the orbital inclination, but there are more satellites in the low-inclination orbit, the overall collision probability is higher, and the collision probability in the working phase in orbit is more than $60 \%$.

When a single satellite is maneuvering and avoiding, it is only necessary to consider the influence of maneuverability and mission execution efficiency. When studying the maneuver avoidance of constellations, it is necessary to comprehensively consider the factors such as the maintenance of constellation configuration, acceptable collision level, avoidance times, etc. This paper focuses on the analysis of the situation when the value of ACPL is $10^{-4}$, during the operation of the satellite, taking reasonable circumvention measures can reduce the risk level by more than $50 \%$. Affected by the number of satellites, the number of avoidance maneuvers in a low-inclination orbital layer is about 1500 and that in a high-inclination orbital layer is about 1500. In practical engineering applications, an efficient collision avoidance method for satellites can be developed according to ACPL values and risk assessment parameters.

The collision of satellite constellations may lead to satellite failure and the increase of space debris. The NASA Break-up model is used to simulate the impact on the space environment after the collision between a single satellite and other mission satellites. On this basis, the Delta debris long-term evolution model and Monte Carlo method are used to evolve the number of space objects in the next 20 years. The number and growth ratio of space objects are analyzed in the case of introducing the Starlink constellation and adopting different PMD success rates for satellites in the constellation. The improvement of the success rate of PMD can alleviate the growth rate of space objects, but cannot offset the impact of the introduction of constellations.

\section{Conclusions}

Launching large constellations into LEO to promote the integration of communication Internet is a development direction that many companies are presently focusing on. In order to ensure the safety and order of the space environment, the relevant responsible personnel need to understand the possible lasting impacts of such missions on the space environment and design them reasonably in the early planning, operation and testing stages. The fewer the number of satellites in the constellation and the smaller the orbital inclination, the less harm to the space debris environment. To realize the function, we can comprehensively consider the relationship between the number of satellites in the constellation and the orbital parameters, and select the scheme that has the least impact on the environment, and achieves the highest reliability of satellite post-mission disposal. It is crucial to strictly comply with the space debris mitigation guidelines proposed by the Inter-Agency Space Debris Coordination Committee (IADC) [22], and create a good space environment order.

Author Contributions: Conceptualization, R.W., X.Y. and S.R.; methodology, S.R., R.W.; software, S.L., R.W. and S.R.; validation, S.R., R.W.; formal analysis, S.R., R.W.; investigation, S.L., X.Y. and X.S.; data curation, S.R.; writing — original draft preparation, S.R.; writing-review and editing, all authors; visualization, S.R., X.S. and S.L. All authors have read and agreed to the published version of the manuscript. 
Funding: This research received no external funding.

Institutional Review Board Statement: Not applicable.

Informed Consent Statement: Not applicable.

Data Availability Statement: This study did not report any data.

Conflicts of Interest: The authors declare no conflict of interest. The funders had no role in the design of the study; in the collection, analyses, or interpretation of data; in the writing of the manuscript, or in the decision to publish the results.

\section{References}

1. Zhao, Q.Y.; Hu, C.B.; Chen, C. Opportunities and challenges of low-orbit large constellations. Space Debris Res. 2020, 1, 1-9.

2. Kessler, D. Collision Cascading: The limits of population growth in low Earth Orbit. Adv. Space Res. 1991, 11, 63-66. [CrossRef]

3. Chun, X. New Development of Oneweb Space Internet Leo Constellation. Satell. Appl. 2016, 6, 75-77.

4. Virgili, B.B.; Dolado, J.C.; Lewis, H.G.; Radtke, J.; Krag, H.; Revelin, B.; Cazaux, C.; Colombo, C.A.; Crowther, R.; Metz, M. Risk to space sustainability from large constellations of satellites. Acta Astronaut. 2016, 126, 154-162. [CrossRef]

5. Virgili, B.B.; Krag, H. Small satellites and the future space debris environment. In Proceedings of the 30th International Symposium on Space Technology and Science, Kobe, Japan, 4-10 July 2015.

6. Virgili, B.B.; Krag, H.; Lewis, H. Mega-constellations and small satellites and their impact on the space debris environment. In Proceedings of the 67th International Astronautical Congres, Guadalajara, Mexico, 27 September 2016.

7. Kitajima, S.; Abe, S.; Hanada, T.; Kawamoto, S. Influences of MEGA constellations on the orbital environment. In Proceedings of the 67th International Astronautical Congres, Guadalajara, Mexico, 27 September 2016.

8. Kawamoto, S.; Hirai, T.; Kitajima, S.; Abe, S.; Hanada, T. Evaluation of space debris mitigation measures using a debris evolutionary model. Trans. Jpn. Soc. Aeronaut. Space Sci. 2018, 16, 599-603. [CrossRef]

9. Lewis, H.; Radtke, J.; Rossi, A.; Beck, J.; Oswald, M.; Anderson, P.; Bastida Virgili, B.; Krag, H. Sensitive of the space debris environment to large constellations and small satellite. J. Br. Interplanet. Soc. 2017, 70, 105-117.

10. Rossi, A.; Alessi, E.M.; Valsecchi, G.B.; Lewis, H.; Radtke, J.; Bombardelli, C.; Bastida Virgili, B. A quantitative evaluation of the environmental impact of the mega constellations. In Proceedings of the 7th European Conference on Space Debris, Darmstadt, Germany, 18-21 April 2017.

11. Space-Track.Org. Available online: www.space-track.org (accessed on 1 July 2021).

12. McDowell, J.C. The Low Earth Orbit Satellite Population and Impacts of the SpaceX Starlink Constellation. Astrophys. J. Lett. 2020, 892, L36. [CrossRef]

13. Chobotov, V.A.; Herman, D.E.; Johnson, C.G. Collision and Debris Hazard Assessment for a Low-Earth-Orbit Space Constellation. J. Spacecr. Rocket. 1997, 2, 233-236. [CrossRef]

14. Radtke, J.; Kebschul, C.; Stoll, E. Interactions of the space debris environment with mega constellations-Using the example of the OneWeb constellation. Acta Astronaut. 2017, 131, 55-68. [CrossRef]

15. Flegel, S.; Gelhaus, J.; Möckel, M.; Wiedemann, C.; Kempf, D.; Krag, H.; Vörsmann, P. Maintenance of the ESA MASTER Model-Final Report 21705/08/D/HK; Institute of Space Systems, TU Braunschweig: Braunschweig, Germany, 2011.

16. Klinkrad, H. Space Debris—Models and Risk Analysis; Springer: Berlin/Heidelberg, Germany, 2006.

17. Space Debris Office. Collision Avoidance Requirements Verification and Guidelines Based on DRAMA/ARES.MIT-COL-MAN-00279OPS-SD; Space Debris Office: Darmstadt, Germany, 2020.

18. ESA/ESOC Space Debris Office. Debris Risk Assessment and Mitigation Analysis (DRAMA) Software User Manual; ESA/ESOC Space Debris Office: Darmstadt, Germany, 2020.

19. Walker, R.; Martin, C.E.; Stokes, P.H.; Wilkinson, J.E.; Klinkrad, H. Analysis of the effectiveness of space debris mitigation measures using the DELTA model. Adv. Space Res. 2001, 28, 1437-1445. [CrossRef]

20. Foreman,V.L.; Siddiqi, A.; Weck, O.L. Large Satellite Constellation Orbital Debris Impacts: Case Studies of OneWeb and SpaceX Proposals. In Proceedings of the AIAA SPACE and Astronautics Forum and Exposition, Orlando, FL, USA, 12-14 September 2017.

21. Johnson, N.L.; Krisko, P.H.; Liou, J.C.; Anz-Meador, P.D. NASA's new breakup model of EVOLVE 4.0. Adv. Space Res. 2001, 28, 1377-1384. [CrossRef]

22. IADC 2007, IADC Space Debris Mitigation Guidelines, IADC-02-01 Revision 1. Available online: www.iadc-home.org/ documents_public (accessed on 15 July 2021). 